\title{
Digging up the dirt on galaxies with Herschel
}

\section{Loretta Dunne ${ }^{1}$}

University of Canterbury

Department of Physics and Astronomy, Private Bag 4800, Christchurch, 8140, New Zealand

E-mail: loretta.dunne@canterbury.ac.nz

Herschel conducted many different surveys of galaxies, both near and far. Herschel's unique strength was that it covered the peak of the dust thermal spectrum, from nearby galaxies out to those at high redshifts. Opening a new observing window in the sub-millimetre, Herschel imaged cool dust in galaxies with higher angular resolution than previously possible and with unprecedented sensitivity. Some Herschel surveys were to study galaxies for certain types in great detail, while others were large area, blank field surveys providing a statistical overview of the quantity of dust and dust obscured star formation over cosmic history. I will review some of the highlights from Herschel about dust in galaxies, covering results on dust scaling relations, dust heating, dust/star geometry, evolution of dust over cosmic time and attenuation in galaxies.

The Life Cycle of Dust in the Universe: Observations, Theory, and Laboratory Experiments (LCDU 2013 ) November 18-22, 2013

Taipei, Taiwan

1

Speaker 


\section{Introduction}

The review task 'What have we learned from Herschel about dust in galaxies?' is a tall order to manage in a $30 \mathrm{~min}$ talk. As a result there is a lot of work in this area that cannot be reviewed here, some of which has been covered already in other talks at this meeting. I thus apologise for the many omissions that are unavoidable since entire conferences have been dedicated to this very question already.

\subsection{What do 'Galaxy People' want from dust?}

Much of the conference has focussed on how we learn about the properties of interstellar dust from observations and laboratory experiments. Turning this around a little, your average extragalactic astronomer is mainly interested in the questions of how galaxies form and evolve, and they use emission from dust to help detect galaxies and understand their properties. Communication between the dust physics (i.e. most people at this meeting) and dust exploitation communities is not always ideal. Top of the wish list for most extragalactic far-infrared astronomers is to have a simple (and accurate) way to relate far-IR emission to star formation rate and dust mass. In many cases, this boils down to fitting a single modified black body to the spectral energy distribution (SED) and using an "off the shelf" value for the dust mass opacity coefficient $(\kappa)$. In communicating the developments in our understanding of the true nature and complexity of dust, it is worth bearing in mind that a section of the community will try to distil this into the most basic form (which may alarm some of the audience!)

\subsection{Herschel's Studies of Galaxies}

The Herschel Space Observatory [1] was launched in 2009 and provided 3.5 years of operations surveying the far-IR and submillimetre skies at unprecedented angular resolution and sensitivity. The two instruments providing imaging were PACS (Photo-conductor Array Camera [2]) and SPIRE (Spectral and Photometric Imaging REceiver [3]), giving 6 bands from 70$500 \mu \mathrm{m}$ and angular resolution from 8-36 arcsecs. Spectroscopy was also provided from 50$600 \mu \mathrm{m}$ by PACS, the SPIRE FTS (Fourier Transform Spectrograph) and HIFI (HerschelHeterodyne Instrument for the Far-Infrared [4]). Herschel conducted a number of surveys of galaxies: some 'blind' to measure statistical properties in an unbiased way, and others targeted on particular samples of galaxies to provide more detail on certain classes of sources. The leaps made in our understanding of dust in galaxies, and about galaxies 'through the medium of dust' have been possible due to Herschel's quite amazing sensitivity and mapping speed (Fig. 1). 


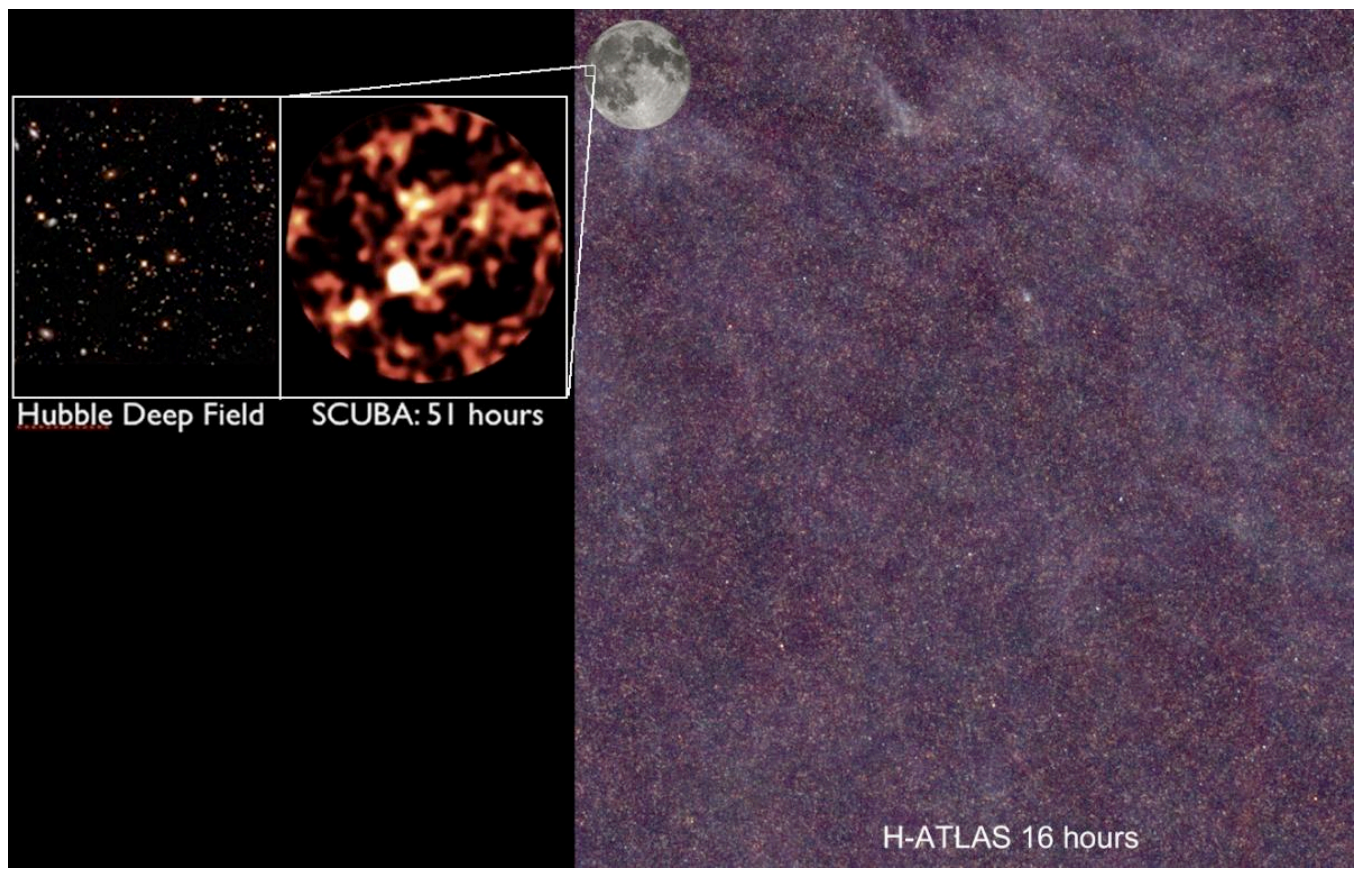

Fig 1. SPIRE 3-colour image of the $16 \mathrm{sq}$ deg Science Demonstration field in HerschelATLAS [5], mapped to the confusion limit in 16 hrs. The full moon is shown for scale and inset is the SCUBA Hubble Deep Field [6] that took 51 hrs with SCUBA to the $850 \mu \mathrm{m}$ confusion limit. This illustrates the kind of wide-area survey made possible by Herschel, which had hitherto been unfeasible at sub-millimetre wavelengths.

\section{Questions for Herschel}

- How do dust properties vary with galaxy type and mass?

- What heats the dust in galaxies, does it trace star formation?

- How is dust distributed relative to stars?

- How much dust is there, and does it evolve?

- How opaque are galaxies?

\subsection{Dust along the Hubble Sequence}

The specific dust mass $\left(\mathrm{M}_{\mathrm{d}} / \mathrm{M}_{\text {star }}\right)$ peaks in galaxies with later types $[7,8]$, and this correlation is also reflected in an anti-correlation between specific dust mass and stellar mass (the 'small ones are more dusty'- [7-9]). The driver for these two relationships appears to be specific star formation rate (sSFR), as small late type galaxies have higher sSFR in the local Universe $[7,8,10,11]$

Herschel has made real breakthroughs in studies of the cold dust content in Early Type Galaxies (E-S0; ETG), both because of its sensitivity to cold dust and its ability to survey wide areas. The blind sub-mm selection made possible through wide area surveys such as HerschelATLAS [5] has revealed a population of ETG which are much dustier than optically selected ETG samples which are targeted with far-IR instruments (Fig. 2a) [10,12]. Targeted surveys 
such as the Herschel Reference Survey (HRS [13]) or KINGFISH [14] find lower average dust content because their ETG samples favour more massive galaxies in higher density environments. Optically selected samples targeted by Herschel find that ETG have slightly warmer dust temperatures than late-type galaxies, which is attributed to the higher interstellar radiation fields (ISRF) in ETGs [7,15]. Dust selected ETG from the wide blank field surveys (H-ATLAS) are more dusty, bluer and reside in lower density environments compared to optically selected ETG (Fig. 2b). They also have younger stellar populations. The dust in these ETG may be the result of a recent merger or may signpost galaxies transitioning from the blue to red sequence $[10,12]$. The relatively shallow depth of Herschel-ATLAS means that only the dustiest ETG are detected, however, ETG on the red sequence are detected in deeper FIR imaging by HRS [7]. They have lower specific dust masses, older stellar populations and live in higher density environments.

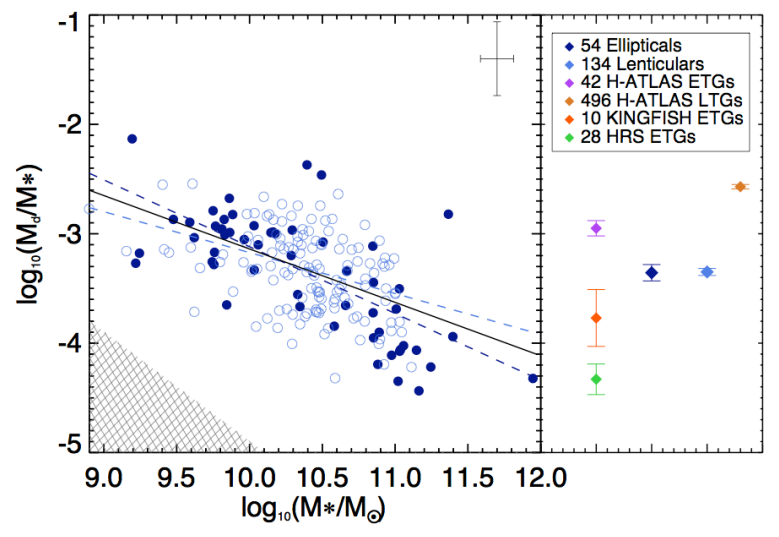

Fig 2a: Relationship of specific dust mass and stellar mass for ETG and specific dust masses for various morphologically selected samples. Taken from $[10]$

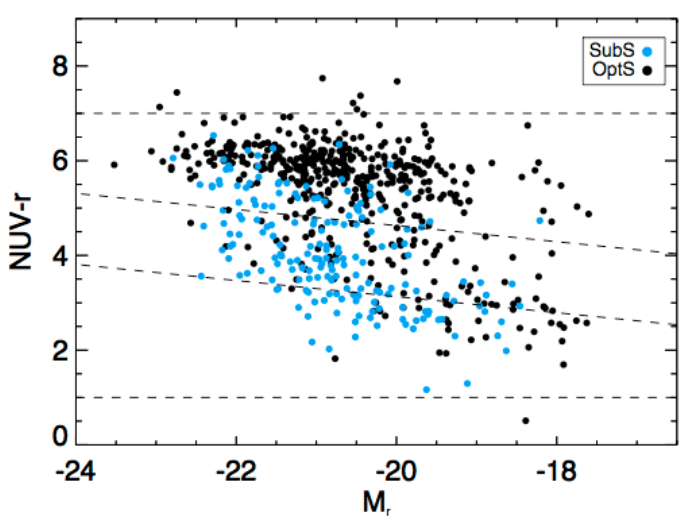

Fig 2b: The striking colour difference between sub-mm detected ETG (blue) and optically selected ETG (black). Taken from [10].

\subsection{Dust Heating}

Dust can be heated in a number of ways within galaxies:

- Old stars (dust in diffuse ISM)

- Young stars in star forming regions (dust in birth clouds)

- UV from star forming regions which escapes (dust in diffuse ISM)

- Active Galactic Nuclei (not considered in this review - but not thought to contribute significantly to the FIR-sub-mm emission of galaxies).

This has been an area of debate in the literature and there is no definitive 'one right answer' to the question of 'what heats the dust in galaxies?' Rather, it depends on the galaxy in question, and in most cases there is a contribution from both old and young stars to powering the FIR/sub-mm emission in a galaxy. 
In spirals, the consensus appears to be that the diffuse component of dust is heated by the older ISRF [13,16-19]. The warmer (70-100 $\mu \mathrm{m}$ emitting) component appears in some cases to be heated by star formation and in others, the older stellar population $[18,20]$. Dust temperatures are generally higher in dwarf galaxies than in their more massive cousins. The dust heating also appears to be better correlated with star formation in dwarfs, which may indicate they have a more porous ISM, allowing UV photons from stellar birth clouds to escape and dominate dust heating in the more massive diffuse component of the ISM $[21,22]$.

One problem in determining the main heating source for dust has been in knowing what to look for. Foyle et al. [23] suggest that $\mathrm{SFR} / \mathrm{M}_{\mathrm{d}}$ is the best quantity to compare to dust temperature in order to look for a correlation between dust heating and young stars (Fig. 3). This quantity is used as a proxy for star formation efficiency (SFE), assuming a reasonable value for gas-to-dust ratio. Simply comparing SFR with dust temperature fails to take account of the mass of dust available to absorb the photons from the SFR.

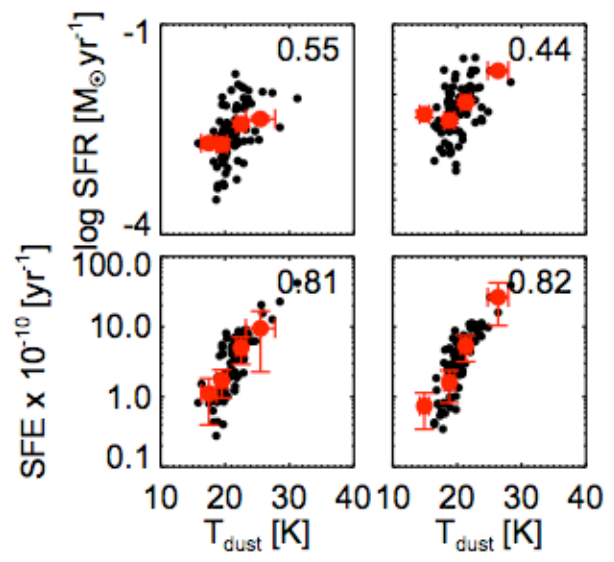

Fig. 3: Correlations between SFR and dust temperature, and SFE (SFR/[M $\left.\left.\mathrm{M}_{d} \times 100\right]\right)$ and dust temperature for FIR knots in M83 [23]. The correlations with SFE are much tighter.

Given the variable contributions of old and young stars to the heating of dust in galaxies, care should be taken when using Herschel data as a measure of star formation. This will only be accurate if appropriate calibrations are used for the galaxy in question (i.e. some account is taken of the amount of un-absorbed UV light and the amount of FIR emission powered by the old stellar ISRF, e.g. [24]).

\subsection{Radiative transfer and dust geometry}

The large photometric range of Herschel, covering the FIR peak and beyond to sample the cold dust, combined with high angular resolution compared to previous FIR missions means that it has provided a wealth of data for radiative transfer studies in local galaxies. Several approaches [25-28] conclude that a second clumpy dust component is required in order to reproduce the FIR emission; while a smooth diffuse dust disk produces the optical-IR extinction, it fails to account for the IR emission by factors of 5-10 (Fig. 4a). Most of the mass of dust is inferred to reside in the colder clumpy component, which has no embedded sources 
(Fig. 4b). There are indications that dust/star geometry may be mass dependent in low redshift galaxies. A study of a low mass disk only spiral (NGC4244) favours a more extended dust distribution relative to stars (dust scale lengths of 2-5 times that of the stars) with dust and stars having similar vertical scale-heights. This suggests that mixing of the dusty ISM within the stellar disk is efficient at low stellar masses and the vertical structure is consistent with that produced by a balance of turbulence and self-gravity [28]. In massive spirals, the dust scale lengths are smaller and the dust is confined to a thin dust lane, this likely will have important consequences for the propagation of optical light in galaxies of different sizes.

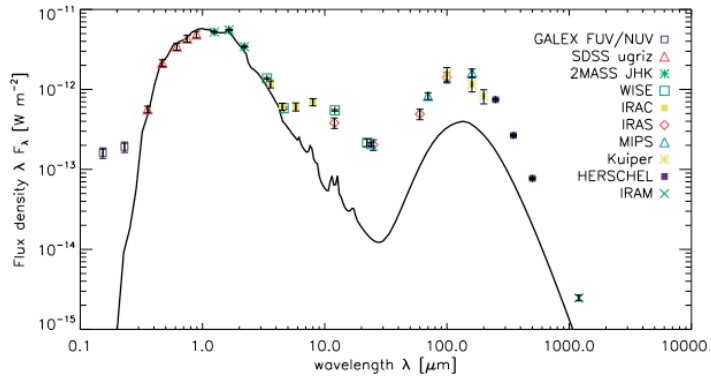

Fig 4a: Model containing old stars and a diffuse dusk disk [27].

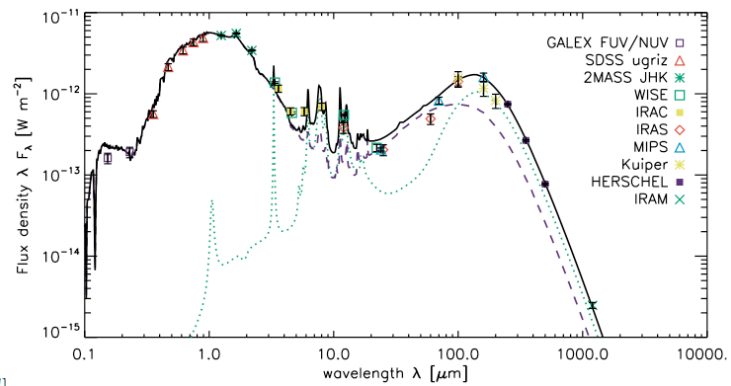

Fig. 4b: Including young stars at $<100 \mathrm{Myr}$ embedded in dusty regions helps produce midIR emission (purple dashed) but to fully reproduce the FIR/sub-mm emission extra quiescent clumps of dust are required (green dotted) [27].

\subsection{Dust content and evolution in galaxies}

Herschel's ability to detect the warm and cold dust in galaxies (hence, with correct modelling, providing a reliable determination of the total dust mass) has revealed that galaxies are dustier than previously thought from surveys using only data at $\lambda<160 \mu \mathrm{m}$ [16]. The huge increase in the number of known dusty galaxies since Herschel has allowed us to trace the evolution of dust content in galaxies over the past 4-5 Gyr by measuring the 'dust mass function' [29]. There has been strong evolution in the dust mass function (Fig 5), and assuming that dust remains a good tracer of molecular gas over this period, this implies that galaxies were both dustier and more gas-rich at $\mathrm{z}=0.5$ compared to today $[29,30]$. This should not be surprising given the decline in the cosmic star formation rate density since $\mathrm{z}=1$, but it is good to see real evidence for changes in ISM content over this interesting period in cosmic history.

Accounting for the increase in dust mass in galaxies has been a challenge for chemical and dust evolution modelling, with efficient grain growth as well as production by Type-II supernovae, and AGB stars required to produce the high dust-to-stellar mass ratios seen in galaxies in Herschel surveys, even at low redshifts out to $z=0.5$ [29] (plus see Rowlands contribution in this volume). 


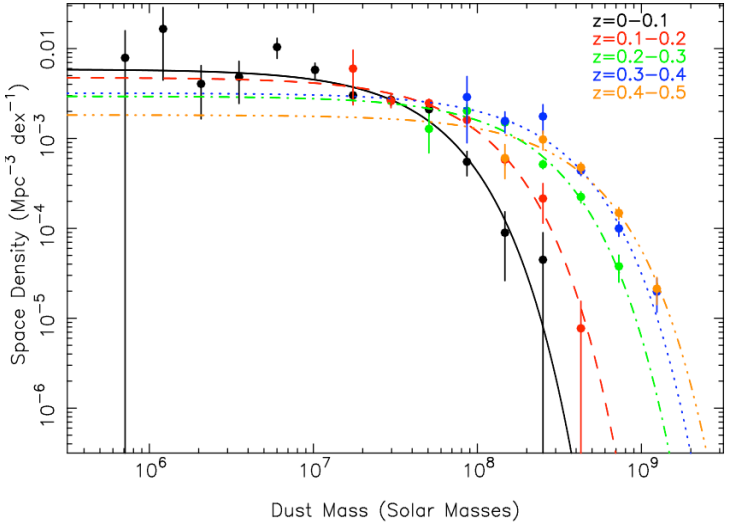

Fig 5a: The dust mass function over the past 5 Gyr of cosmic history from [29]. Galaxies had higher characteristic dust masses in the past, consistent with an increase in their overall ISM content.

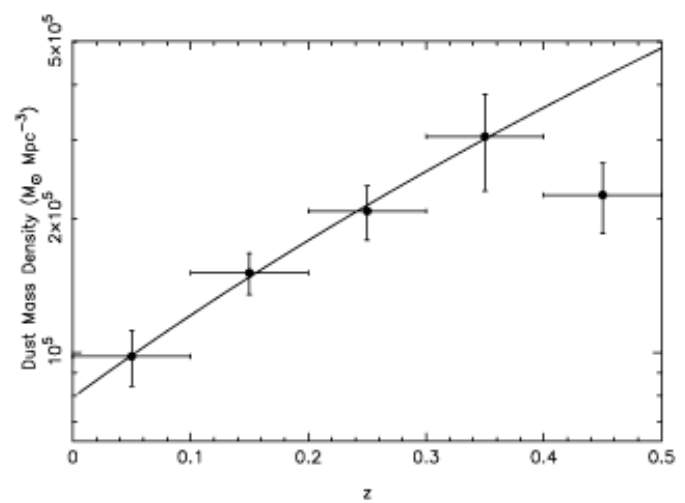

Fig 5b: The evolution is cosmic dust mass density from [29]. The turn down at $z>0.4$ is a result of incompleteness, and should be resolved in future when deeper optical data is available for matching to the Herschel sources.

\subsection{The evolution of galaxy opacity}

By combining UV data with Herschel data, it is possible to measure the attenuation in the UV $\left(\mathrm{A}_{\mathrm{UV}}\right)$ directly. Using deep Herschel surveys [31,32] that probe galaxies across a large range of cosmic time, a picture of the evolution of attenuation in galaxies can be built up $([33,34]$, Greve et al. in prep). The peak dust attenuation occurs at $\mathrm{z}=1.2$ and then declines towards today (Fig. 6a). Interestingly, the total star formation rate can also be computed from $\mathrm{L}_{\mathrm{UV}}+\mathrm{L}_{\mathrm{IR}}$, and it can be seen that the peak in dust attenuation $\left(\mathrm{A}_{\mathrm{UV}}\right)$ lags the star formation activity [33]. There is currently no clear explanation for this recent finding, but there may be interesting links with timescales for dust formation/growth or spatial movements within the ISM (Fig. 6b).

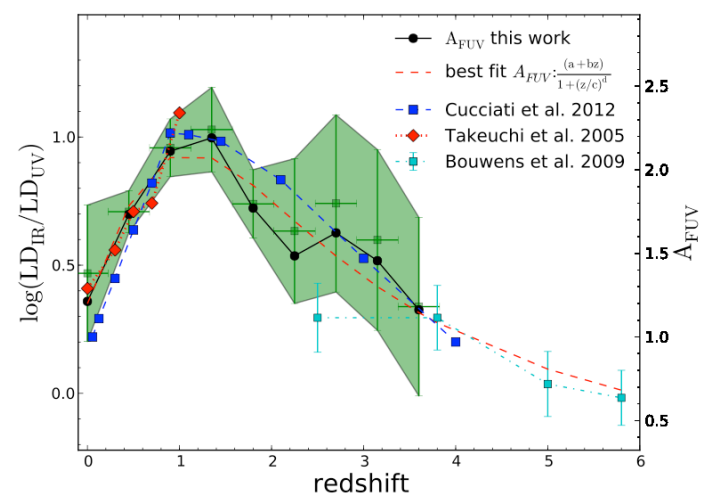

Fig 6a: The evolution of $A_{U V}$, showing a peak at $\mathrm{z} \sim 1.2$ (taken from [33])

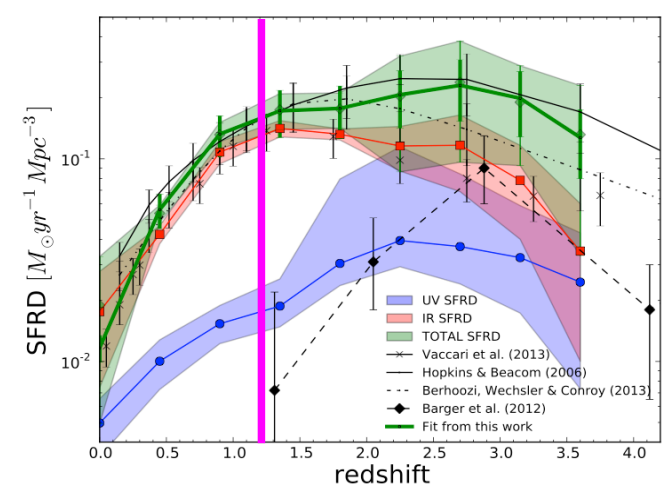

Fig 6b: Compilation of the evolution of the cosmic SFRD, showing a broad peak from $\mathrm{z}=1-3$. The position of the peak in $A_{U V}$ is overlaid as a magenta line. Adapted from [33]. 


\subsection{Probing dust properties using the UV attenuation law.}

The relationship between UV spectral slope $(\beta)$ and the attenuation (called IR excess or IRX: $\mathrm{L}_{\mathrm{IR}} / \mathrm{L}_{\mathrm{UV}}$ ) has been widely used to correct UV studies for the affects of dust. The calibration for this relationship was based originally on a sample of local starburst galaxies [35] and applied to other high redshift samples on the assumption that they would have similar properties. For UV-selected samples with a limited range in luminosity (around $\mathrm{L}^{*}$ ), this has seemed generally to be the case. However, the most luminous UV sources at $z>4$ and very young galaxies $(<100$ Myr) seem to depart from this relationship suggesting changes in dust covering factor or grain size distribution [36-38]. Herschel has progressed these studies by providing the extremely sensitive FIR observations required to measure $\mathrm{L}_{\mathrm{IR}}$ in UV-selected galaxies (i.e those with 'normal' or 'low' obscuration) at the highest redshifts $(\mathrm{z}=4-5)$.

Using UV properties alone to measure dust attenuation is risky, and relies on having very good awareness of the selection biases inherent in a given study. For example, it is well known that IR-selected samples tend to preferentially select the more obscured galaxies and hence find higher $A_{U V}$ for a given UV spectral slope compared to UV-selected samples $[39,40]$. Comparing LIRGS (Luminous Infrared Galaxies with $\mathrm{L}_{\mathrm{IR}}>10^{11} \mathrm{~L}_{\mathrm{sun}}$ ) at low and high redshift does, however, show that $\mathrm{z}=2$ LIRGs are more transparent to UV radiation (i.e. have lower $\mathrm{L}_{\mathrm{IR}} / \mathrm{L}_{\mathrm{UV}}$ ) than LIRGs in the local Universe [40]. This may indicate that the conditions required to produce LIRG luminosities are less 'extreme' at high redshift, i.e. the star formation events can be more wide-spread and less obscured and still produce the same luminosity.

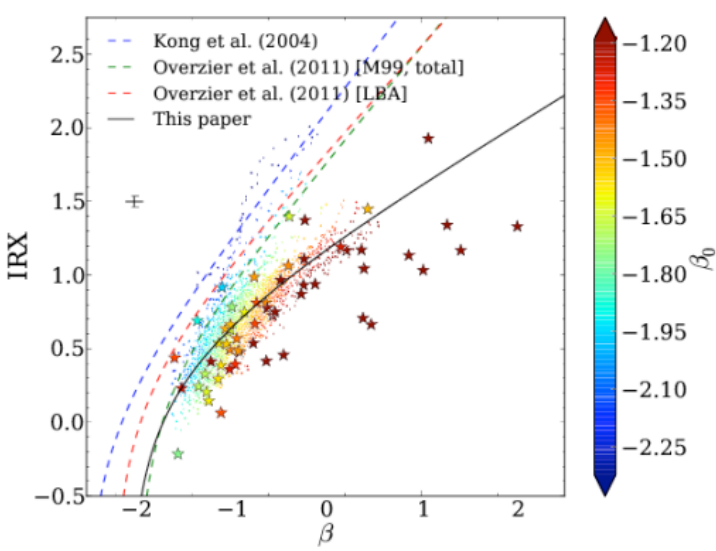

Fig. 7a: IR excess against UV spectral slope for galaxies from the Herschel Reference Survey [42]. The relationship between attenuation and UV spectral slope is different for normal star forming galaxies and starbursts (shown by the dashed lines). Stars represent individual galaxies from HRS, small points are resolved regions within galaxies. Points are colour coded according to their intrinsic spectral slope (stellar population dependent), which is derived from fitting to the broadband spectral energy distributions [42].

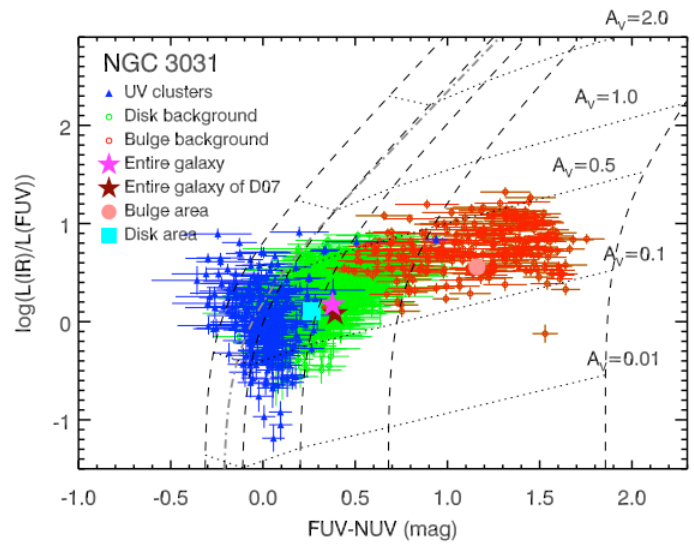

Fig. 7b: IR excess vs. UV colour for regions within a single galaxy from [43]. The changes in UV colour for regions of increasing stellar age are apparent - from young UV clusters (blue) to old stars in the bulge (red). The dashed lines show model curves for ages between 2-500 Myr while the dotted lines connect these at constant $A_{V}$. This highlights the problem in using FUV-NUV and a fixed assumption about age to estimate attenuation. 
There are now known to be issues in applying the UV/IRX relation to galaxies with a range of stellar histories (as opposed to those defined in a specific 'starburst' phase), as many studies have shown that normal galaxies do not lie on the same relation as starbursts [e.g. 41]. This brings into question its widespread use as a method by which to correct UV-derived star formation rates at higher redshifts. The problem is that the UV slope is sensitive to the age of the stellar populations as well as the dust attenuation (which in turn depends on dust properties and geometry).

Resolved studies of local galaxies are now attempting to disentangle the effects of stellar population and dust attenuation in the UV-slope vs. attenuation plane (Fig 7). This is a new approach which at the least holds promise for 'keeping us honest' in dealing with the uncertainties inherent in this method, however, there is also hope that it may reveal trends of IRX/ $\beta$ with dust properties which could be used to probe the type of dust present at high redshifts $([42,43]$, Mao et al. in prep).

\section{References}

[1] Pilbratt G. et al. 2010, The Herschel Space Observatory - An ESA facility for far-infrared and submillimetre astronomy, A\&A, 518, L1

[2] Poglistch A. et al. 2010, The Photodetector Array Camera and Spectrometer (PACS) on the Herschel Space Observatory, A\&A, 518, L2

[3] Griffin M. et al. 2010, The Herschel SPIRE instrument and its in-flight performance, A\&A, 518, L3

[4] De Graauw Th. et al. The Herschel-Heterodyne Instrument for the Far-Infrared (HIFI), A\&A, 518, L6

[5] Eales S. et al. 2010, The Herschel ATLAS, PASP, 122, 499

[6] Hughes D. et al. 1998, High-redshift star formation in the Hubble Deep Field revealed by a submillimetre-wavelength survey, Nature, 394, 241

[7] Smith M. W. L. et al. 2012, The Herschel Reference Survey: Dust in Early-type galaxies and across the Hubble sequence, ApJ, 748, 123

[8] Cortese L. et al., 2012, The dust scaling relations of the Herschel Reference Survey, A\&A, 540, 52

[9] Bourne N. et al. 2012, Herschel-ATLAS/GAMA: A census of dust in optically selected galaxies from stacking at submillimetre wavelengths, MNRAS, 421, 3027

[10] Aigus N. et al. 2013, GAMA/H-ATLAS: linking the properties of submm detected and undetected galaxies - I. $z<0.06$ sample, MNRAS, 431, 1929

[11] Da Cunha E., Eminian C., Charlot S., Blaizot J., 2010, New insight into the relation between star formation activity and dust content in galaxies, MNRAS, 403, 1894

[12] Rowlands K. et al. 2012, Herschel-ATLAS/GAMA: dusty early type galaxies and passive spirals, MNRAS, 419, 2545

[13] Boselli A. et al. 2010, The Herschel Reference Survey, PASP, 122, 261 
[14] Kennicutt R. et al., 2011, KINGFISH - Key Insights on Nearby Galaxies: A Far-Infrared Survey with Herschel: Survey Description and Image Atlas, PASP, 123, 1347

[15] Skibba R. et al. 2011, The emission by dust and stars of nearby galaxies in the Herschel KINGFISH survey, ApJ, 738, 89

[16] Dale D. et al. 2012, Herschel Far-Infrared and Submillimetre photometry for the KINGFISH sample of nearby galaxies, ApJ, 745, 95

[17] Bendo G. et al. 2012, Investigations of dust heating in M81, M83 and NGC2403 with the Herschel Space Observatory, MNRAS, 419, 1833

[18] Boquien M. et al. 2011, Dust Heating Sources in Galaxies: The Case of M33 (HERM33ES), AJ, 142,111

[19] Bendo G. et al. 2010, The JCMT Nearby Galaxies Legacy Survey - III. Comparisons of cold dust, polycyclic aromatic hydrocarbons, molecular gas and atomic gas in NGC 2403, MNRAS, 402, 1409

[20] Li Y. et al. 2013, Star formation rates in resolved galaxies: calibrations with near and far-infrared data for NGC 5055 and NGC 6946, ApJ, 768, 180

[21] Galametz M. et al. 2011, Probing the dust properties of galaxies up to submillimetre wavelengths, II. Dust-to-gas mass ratio trends with metallicity and the submm excess in dwarf galaxies, A\&A, 532,56

[22] Remy-Ruyer A. et al. 2013, Revealing the cold dust in low-metallicity environments. I. Photometry analysis $f$ the Dwarf Galaxy Survey with Herschel, A\&A, 557, 95

[23] Foyle K. et al. 2013, Star formation and dust heating in the FIR bright sources of M83, MNRAS, 434,2182

[24] Kennicutt R. et al. 2009, Dust-corrected star formation rates of galaxies. I. Combinations of Ho and Infrared tracers, ApJ, 703, 1672

[25] Baes M. et al. 2010, Herschel-ATLAS: The dust energy balance in the edge-on spiral galaxy UGC4754, A\&A, 518, L39

[26] Bianchi S. et al. 2008, Dust extinction and emission in a clumpy galactic disk. An application of the radiative transfer code TRADING, A\&A, 490, 461

[27] De Looze I. et al. 2012, The dust energy balance in the edge-on spiral galaxy NGC4565, MNRAS, 427,2797

[28] Holwerda B. et al. 2012, Herschel/SPIRE observations of the dusty disk of NGC4244, A\&A, 541, L5

[29] Dunne L. et al. 2011, Herschel-ATLAS: rapid evolution of dust in galaxies over the last 5 billion years, MNRAS, 417, 1510

[30] Berta S. et al. 2013, Molecular gas mass functions of normal star-forming galaxies since $z \sim 3$, A\&A, 555, L8

[31] Lutz D. et al. 2011, PACS Evolutionary Probe (PEP) - A Herschel Key Program, A\&A, 532, 90

[32] Oliver S. et al. 2012, The Herschel Multi-tiered Extragalactic Survey: HerMES, MNRAS, 424, 1614 
[33] Burgarella D. et al. 2013, Herschel PEP/HerMES: the redshift evolution $(0<z<4)$ of dust attenuation and of the total $(U V+I R)$ star formation rate density, A\&A, 554, 70

[34] Cucciati O. et al. 2012, The star formation rate density and dust attenuation evolution over 12 Gyr with the VVDS survey, A\&A, 539, 31

[35] Meurer G. et al. 1999, Dust absorption and the ultraviolet luminosity density at $z \sim 3$ as calibrated by local starburst galaxies, ApJ, 521, 64

[36] Reddy N. et al. 2006, Star formation and extinction in redshift z 2 galaxies: Inferences from Spitzer MIPS observations, ApJ, 644, 792

[37] Siana B. et al. 2009, Detection of Far-Infrared and polycyclic hydrocarbon emission from the cosmic eye: probing the dust and star formation of Lyman Break Galaxies, ApJ, 698, 1273

[38] Lee K. et al. 2012, Herschel detection of dust emission from UV-luminous star-forming galaxies at $3.3<z<4.3$, ApJ, 758, L31

[39] Oteo I. et al. 2013, Far-infrared detected Lyman-break galaxies at z 3. Dust attenuation and dust correction factors at high redshift, A\&A, 554, L3

[40] Reddy N. et al. 2012, GOODS-Herschel measurements of the dust attenuation of typical star forming galaxies at high redshift: observations of ultraviolet-selected galaxies at $z \sim 2$, ApJ, 744, 154

[41] Kong X. et al. 2004, Star formation history and dust content of galaxies drawn from ultraviolet surveys, MNRAS, 349, 769

[42] Boquien M. et al. 2012, The IRX- $\beta$ relation on subgalactic scales in star-forming galaxies of the Herschel Reference Survey, A\&A, 539, 145

[43] Mao Y. et al. 2012, Characterizing ultraviolet and infrared observational properties for galaxies. I. Influences of dust attenuation and stellar population age, ApJ, 757, 52 